\title{
Reconstruction of Juxta-Articular Bone Defect in an Infected Fracture Distal Right Femur Using Autologous Fibular Strut and Iliac Crest Graft
}

\author{
Nareshkumar Satyanarayan Dhaniwala1, Khizar Khusrau Khan², Salahuddin Ahmed ${ }^{3}$ \\ 1, 2, 3 Department of Orthopaedics, Jawaharlal Nehru Medical College, DMIMS, Sawangi, Wardha, Maharashtra, India.
}

\section{INTRODUCTION}

Distal femur fractures are about $7 \%$ of all femur fractures. ${ }^{1}$ Being complex in nature management of these fractures is difficult; management is still a challenge in this technically advanced era. Fibular strut graft is one of the useful and simple options to manage defects in distal femur fractures. High velocity injuries causing open fractures may lead to infection and non-union of fracture if proper debridement and antibiotics are not used as per established protocol. Antibiotics can be given mixed with polymethyl-methacrylate (PMMA) cement formed in beads at the open fracture site or coated over a nail. ${ }^{2}$ This causes slow release of antibiotics and helps in control of infection. Local infection, damaged soft tissue, ischemia over fracture site, stabilisation of fracture, the hardware used and patient's co morbidities all play a role in union and infection control of open fractures. Early detection of infection is important to avoid non-union and related complications.

Distal femur juxta-articular fracture may develop infection due to fractures and unstable fixation more commonly. This may result in cavity formation and complications like osteomyelitis often leading to bone destruction and sequestrum formation. Its management becomes a challenging task for an orthopaedic surgeon. ${ }^{3-}$ 9 The reconstructive methods are autogenous cortical bone, cancellous bone, allograft, bone substitutes like synthetic bone blocks and bone granules. Free bone transfer is crucial in reconstructing massive defects in distal femur fracture due to properties of rapid healing and being hypertrophic. ${ }^{7-13}$ Fibula is the best available option for grafting in massive defects of femur due to its characteristics like mechanical strength, length, minimum morbidity, and resistance to infection. . $^{311,13} \mathrm{~A}$ single fibula strut graft can give up to $26 \mathrm{~cm}$ of graft. The case reported here is of a patient of juxta-articular bone defect in an infected previously operated fracture distal femur using autologous fibular strut and iliac crest graft.
Corresponding Author: Dr. Salahuddin Ahmed, Raghabaji Boys Hostel, JNMC Campus, DMIMS, Sawangi, Wardha - 442001,

Maharashtra, India.

E-mail: Salahuddin1810@gmail.com

DOI: $10.14260 /$ jemds/2021/709

How to Cite This Article:

Dhaniwala NS, Khan KK, Ahmed $S$, Reconstruction of juxta-articular bone defect in an infected fracture distal right femur using autologous fibular strut and iliac crest graft. J Evolution Med Dent Sci 2021;10(39):3501-3504, DOI: 10.14260/jemds/2021/709

Submission 12-03-2021,

Peer Review 04-07-2021,

Acceptance 11-07-2021,

Published 27-09-2021.

Copyright (c) 2021 Nareshkumar Satyanarayan Dhaniwala et al. This is an open access article distributed under Creative Commons Attribution License [Attribution 4.0 International (CC BY 4.0)] 


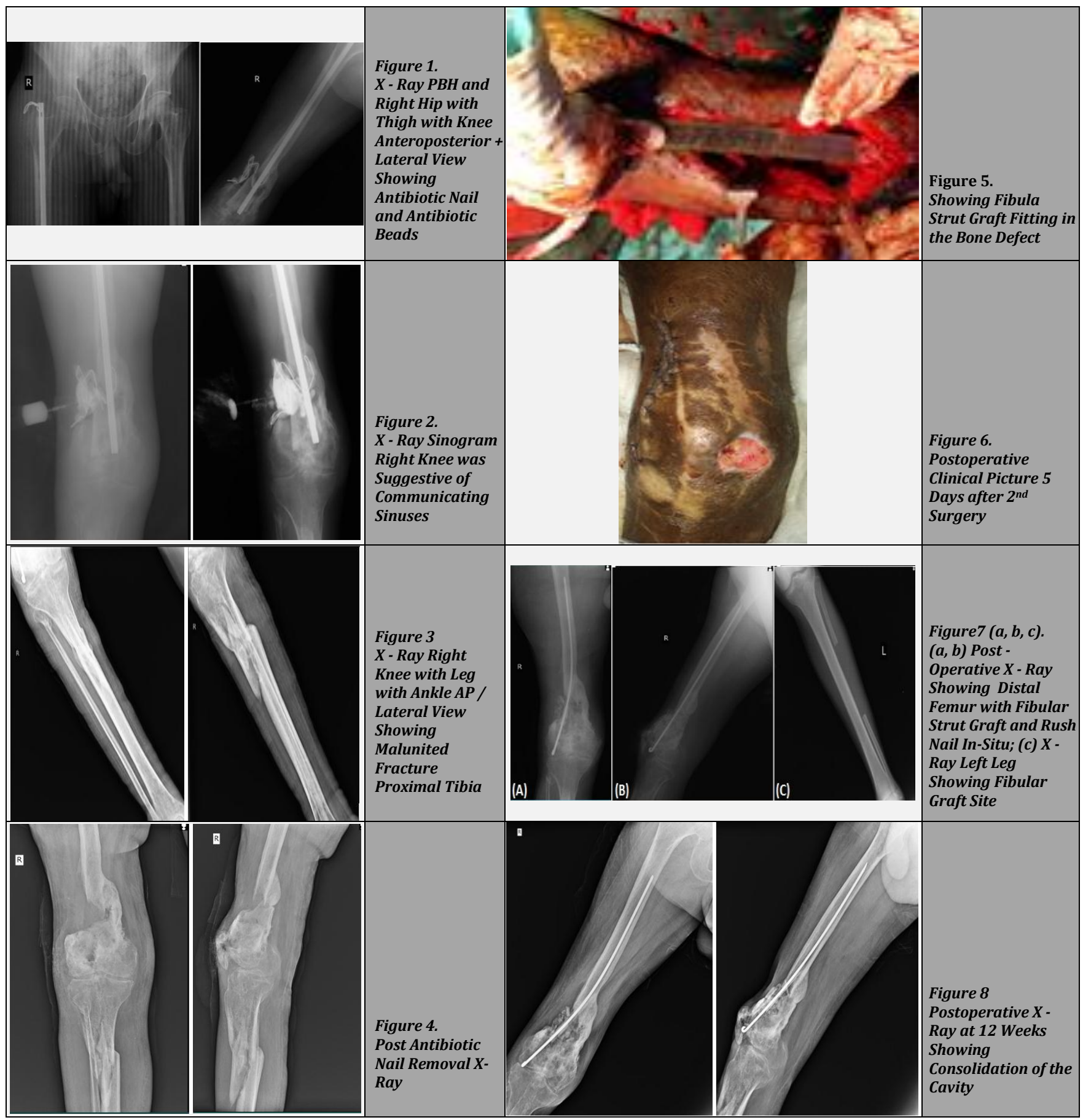

\section{PRESENTATION OF CASE}

A 41 - year - old patient, farmer by occupation presented in orthopaedic OPD with stiffness, pain and purulent discharge over right knee since 2 months. Patient gave a history of operated right compound distal femur fracture trauma one and half year back. He was managed by wound debridement, antibiotic nailing and plaster for leg bones. Patient was asymptomatic for 1 year and later he developed 3 purulent discharging sinuses in lower part of the right thigh with intermittent fever over the last 3 months. He gave history of similar discharges 1 year back which had subsided after regular dressing. Getting no relief by repeated dressings he consulted our centre. Examination revealed irregularity of lower third shaft femur with three discharging sinuses which were fixed to the underlying bone. There were wounds of $3 \mathrm{x}$ $3 \mathrm{~cm}$ and $2 \times 1 \mathrm{~cm}$ size proximal to patella and on the lateral aspect of lower thigh size on anterior thigh. There were surgical scars indicating intramedullary nailing. Knee movement was grossly restricted. Patient was unable to put weight on the right lower limb. He had a comminuted oblique fracture at the upper third shaft right tibia which was united clinicoradiologically.

$\mathrm{X}$ rays and haematological investigations were done to evaluate the pathology. X - Ray of pelvis and thigh (Figure 1) showed an old comminuted fracture at the lower third right femur treated with Kuntscher nail along with antibiotic beads. There was lysis at the fracture site and nail was not providing stable fixation. Nail entry was more medial and had a wire loop tied through its eye. Sinogram (Figure 2) showed continuity of sinus to the cavity in lower femur. Mal-united comminuted 
fracture proximal tibia was seen on X - Ray right knee with leg (Figure 3) with ankle anteroposterior and lateral view.

\section{DISCUSSION}

In view of the fracture in non-union with frank infection, antibiotic beads and nails were removed and thorough curettage was done at the infection site. Pre-operatively there was cavity formation in the lower part of femur with loss of bone on anterolateral aspect. There was no obvious fracture in the shaft. The wound was managed using vacuum assisted closure [VAC] and antibiotics were given as per sensitivity (klebsiella sensitive to amikacin and ciprofloxacin). The wound became healthy without any discharge in 10 days. Cultures of discharge taken twice showed no growth. Post Nail Removal X - ray (Figure 4) showed a gap in lower third femur with bridge of bone uniting the fracture postero medially with moderate callus. The second surgery consisted of taking a long piece of opposite fibular bone graft and corticocancellous bone grafts from contralateral iliac crest. The cavity in lower femur was thoroughly curetted and a long piece of fibula strut was used as a bridge between the lower femur and subarticular area. It was further stabilised using a Rush nail (Figure 5). Corticocancellous grafts were used to cover the remaining part of cavity along with remaining fibula cut into small pieces. Post operatively IV antibiotics were continued for 2 weeks. Wound healed as expected lines except a small area on anteromedial aspect which healed slowly (Figure 6). The fixation was adequate and stable filling the gap as evident on the post op $\mathrm{x}$ - ray (Figure 7). The limb was immobilised in long knee brace and non-weight bearing ambulation with walker was permitted after suture removal. Active knee mobilisation was encouraged. He had 0 - 25 degree movement at knee 3 weeks post-surgery. At 12 weeks' postoperatively patient was asymptomatic. He was walking with support of stick and had knee range of movement of $0-30$ degrees only. His X-ray (Figure 8) showed consolidation progressing in the cavity

Pre-operatively itself the patient was started on muscle strengthening static exercises for quadriceps, hamstrings, and gluteal muscle groups. Post-operatively after 2 weeks these were converted to dynamic exercises, knee ROM of $0-15$ degrees was achieved. Patient was allowed with partial weight bearing mobilization with walker support after 3 weeks. Patient was allowed bearing as tolerated after 4 weeks of surgery. The physiotherapy and mobilization were continued as advised earlier. The patient was followed up after 6 weeks. He was comfortable, with significant pain relief, and was complying well with the physiotherapy regime with knee ROM of 0 - 25 degrees. At 10 months' follow-up patient remained asymptomatic. His knee range of movement had improved to 0 - 40 degrees.

Distal femur juxta-articular fracture with complications like osteomyelitis which results in bone loss due to sequestrum formation are challenging tasks for orthopaedic surgeon. The reconstructive methods like autogenous iliac crest bone grafts and allograft are not enough for reconstruction..$^{3-9}$ Fibula has proved to be a good source of bone graft having strength and length due to its anatomical characteristics. Besides it also has osteogenic and osteoconductive potential as proved in many studies. As against this while using antibiotic impregnated nails, strict postoperative radiographic monitoring is mandatory, as this may result in underlying slow growing infection which goes undiagnosed and presents after extensive damage. Fibular graft has been used in many studies. Tani et al. used fibular graft to bridge the gap in a fracture case, the graft extending from medullary cavity to intercondylar area of femur. ${ }^{14}$ Ramesh et al. treated 13 patients having distal femoral fractures type C3, with corticocancellous and fibular graft, stabilized with ring fixator. ${ }^{15}$ Lawal et al. used free fibular graft to treat defects in open fractures of femur and tibia and reported incorporation in more than $2 / 3^{\text {rd }}$ cases. ${ }^{16}$ Yajima et al. treated 20 non-union cases with vascularized free fibular graft. Most of his patients united well. Muramatsu et al. treated 17 post traumatic / post tumour defects in femur using vascularized fibular graft and noted good outcome in $93 \%$ cases. ${ }^{17}$ Yajima et al. and Muramatsu et al. both noted graft fracture in 3 cases each. ${ }^{17}$ In the present case, an autogenous fibular strut graft along with iliac crest corticocancellous graft, has been used to manage bone defect. The fibular strut extended from medulla of the lower third femur up to subarticular region of lateral condyle. Graft provided length maintenance, osteogenicity and stability. Gradual radiological union was seen with control of infection

The fixation method used in this case by rush nail provides splintage and stability to retain the graft in position. Graft size is required to be optimum for maintaining length of the bone and stability at both its ends. Rush nail provides alignment to the graft besides working as a means of moderate fixation. Fibular graft can be seen to hypertrophy after union at its ends and may gain strength over a period of time. Delayed weight bearing may help to reduce the risk of postoperative stress fracture. Long term follow-up is needed to note further progress and any complications. This procedure is relatively simple and can be done without any special expertise.

\section{CONCLUSIONS}

Fibular strut grafting is a technically simple and useful method to fill in gaps in fracture and non-union cases due to its inherent strength and osteogenic potential.

Conflict of interest: none

Financial or other competing interests: None.

Disclosure forms provided by the authors are available with the full text of this article at jemds.com.

\section{REFERENCES}

[1] Arneson TJ, Melton LJ, Lewallen DG, et al. Epidemiology of diaphyseal and distal femoral fractures in Rochester, Minnesota, 1965-1984. Clin Orthop Relat Res 1988;234:188-94.

[2] Zalavras CG, Patzakis MJ, Holtom P. Local antibiotic therapy in the treatment of open fractures and osteomyelitis. Clin Orthop Relat Res (1976-2007) 2004;427:86-93.

[3] Hou SM, Liu TK. Reconstruction of skeletal defects in the femur with 'two-strut' free vascularized fibular grafts. J Trauma 1992;33(6):840-5. 
[4] Yajima H, Tamai S. Twin-barrelled vascularized fibular grafting to the pelvis and lower extremity. Clin Orthop Relat Res 1994;303:178-84.

[5] Chen MT, Chang MC, Chen CM, et al. Double-strut free vascular fibular grafting for reconstruction of the lower extremities. Injury 2003;34(10):763-9.

[6] Yajima H, Tamai SU, Mizumoto SH, et al. Vascularised fibular grafts for reconstruction of the femur. J Bone Joint Surg 1993;75(1):123-8.

[7] Yajima H, Tamai S, Mizumoto S, et al. Vascularized fibular grafts in the treatment of osteomyelitis and infected nonunion. Clin Orthop Relat Res 1993;293:256-64.

[8] Lai D, Chen CM, Chiu FY, et al. Reconstruction of juxtaarticular huge defects of distal femur with vascularized fibular bone graft and Ilizarov's distraction osteogenesis. J Trauma 2007;62(1):166-73.

[9] Liang K, Cen S, Xiang Z, et al. Massive juxta-articular defects of the distal femur reconstructed by series connected double-strut free-vascularized fibular grafts. J Trauma Acute Care Surg 2012;72(2):E71-6.

[10] Weiland AJ, Phillips TW, Randolph MA. Bone grafts: a radiologic, histologic and biomechanical model comparing autografts, allografts and free vascularized bone grafts. Plast Reconstr Surg 1984;74(3):368-79.
[11] Jupiter JB, Bour CJ, May JW Jr. The reconstruction of defects in the femoral shaft with vascularized transfers of fibular bone. J Bone Joint Surg Am 1987;69(3):365-74.

[12] Malizos KN, Zalavras CG, Soucacos PN, et al. Free vascularized fibular grafts for reconstruction of skeletal defects. J Am Acad Orthop Surg 2004;12(5):360-9.

[13] El-Gammal TA, El-Sayed AM, Kotb MM. Hypertrophy after free vascularized fibular transfer to the lower limb. Microsurg 2002;22(8):367-70.

[14] Tani Y, Inoue $\mathrm{K}$, Kaneko $\mathrm{H}$, et al. Intramedullary fibular graft for supracondylar fracture of the femur following total knee arthroplasty. Arch Orthop Trauma Surg 1998;117(1-2):103-4

[15] Ramesh LJ, Rajkumar SA, Rajendra R, et al. Ilizarov ring fixation and fibular strut grafting for C3 distal femoral fractures. J Orthop Surg (Hong Kong) 2004;12(1):91-5.

[16] Lawal YZ, Garba ES, Ogirima MO, et al. Use of nonvascularized autologous fibula strut graft in the treatment of segmental bone loss. Ann Afr Med 2011;10(1):25-8.

[17] Muramatsu K, Ihara K, Shigetomi M, et al. Femoral reconstruction by single, folded or double free vascularised fibular grafts. $\mathrm{Br} J$ Plast Surg 2004;57(6):550-5. 